\title{
Supramolecular perspectives in colloid science
}

\author{
M. A. Cohen Stuart
}

Received: 13 February 2008 /Revised: 29 February 2008 / Accepted: 3 March 2008 / Published online: 12 April 2008

(C) The Author(s) 2008

\begin{abstract}
Supramolecular chemistry puts emphasis on molecular assemblies held together by non-covalent bonds. As such, it is very close in spirit to colloid science which also focuses on objects which are small, but beyond the molecular scale, and for which other forces than covalent bonds are crucial. We discuss in this review the preparation and properties of new colloidal systems which borrow on the one hand from classical topics in colloid science, such as micellization, and on the other hand from concepts in supramolecular chemistry, such as reversible supramolecular polymers.
\end{abstract}

Keywords Coacervation · Micellization ·

Supramolecular polymers

\section{Introduction}

Chemistry is concerned with the question how atoms combine into structures that eventually become materials with certain properties and functions. In particular, synthetic organic chemistry developed into a highly refined art to make molecules, multi-atomic particles held together by covalent bonds. However, one molecule does not yet make a material. Between molecules, weaker, non-covalent interactions play their part in defining the structure and dynamics of systems. Employing such interactions to make molecular assemblies with a significant lifetime, so that one can see

M. A. Cohen Stuart $(\square)$

Laboratory of Physical Chemistry \& Colloid Science, Wageningen University,

PO Box 8038, 6700 EKWageningen, The Netherlands

e-mail: martien.cohenstuart@wur.nl them as a kind of 'giant molecule', might be considered as an extension of classical synthetic chemistry into the realm of 'supermolecules'. Hence the term 'supramolecular chemistry' was coined by Jean-Marie Lehn to denote this particular scientific endeavor [1]. Supramolecular chemistry has met enormous enthusiasm; it is almost the latest paradigm in synthetic chemistry.

How does this relate to colloid science? Colloidal systems are often composed of multimolecular particles as well, e.g., emulsion droplets or latex particles consist of many molecules which stay together simply because they are insoluble in the continuous phase. However, these particles do not really qualify as supramolecular assemblies in the sense alluded to above. A different class of colloidal systems is that of the association colloids, in which molecules reversibly associate into multimolecular particles with a well-defined size and structure. The classical example is that of soap micelles: the amphiphilic soap (surfactant) molecules stay together because they have an insoluble part ('tail') which tends to avoid contact with the solvent, but they also have a soluble moiety ('head') which has to remain well solvated. The compromise is a structure which shields the tails from the solvent so that its area per unit mass is large, and this implies either small particles or thin threads (spherical or cylindrical micelles) or thin sheets (lamellae), or hybrids between these (bicontinuous structures). In a way, surfactant micelles, because they have a (thermodynamically) defined size and average shape, can be considered as supramolecular entities. Since they organize themselves spontaneously, they are sometimes referred to as 'self-organised nanosystems'. Micellar systems have since long been studied extensively within the colloid science community [2], so that it is not too farfetched to say that colloid scientists were involved in supramolecular chemistry 'avant la lettre'. 
A more interesting question is: can we find inspiration from supramolecular chemistry to develop colloid science? I think we certainly can, because (a) supramolecular chemistry can provide us with new kinds of colloidal objects and because (b) supramolecular objects can induce new kinds of surface forces. In the remainder of this review, I will discuss examples illustrating this claim.

\section{Thermodynamic picture of micellization}

Let us first return to our classical example: the soap micelle. Such a particle forms by spontaneous aggregation of amphiphilic objects, e.g., molecules with one soluble and one insoluble part, see Fig. 1. The primary driving force for the aggregation is provided by the insoluble part: for each free molecule transferred to the aggregate's interior the free energy of solvation drops by a certain amount ('volume term'). However, a small aggregate does not have so much interior: a substantial fraction of the molecules is still in contact with the solvent rather than with molecules of its own kind. Hence, a positive correction to the free energy must be introduced, the importance of which obviously scales with the surface area of the aggregate ('surface tension term'). The sum of these two free energies varies non-monotonically with the aggregates' size: first it goes up because the surface term dominates; at larger sizes, it decreases because the volume term becomes more important.

If this were the whole picture, particles, once formed, would tend to grow to very large sizes (because the free energy would continue to decrease with size). In fact the two terms just mentioned are the ingredients of classical nucleation theory [3]. However, free growth is prohibited

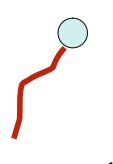

$\mathrm{n}_{\mathrm{m}}=1$

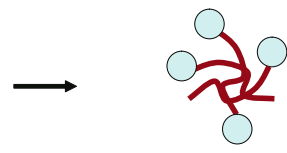

$\mathrm{n}_{\mathrm{m}}=4$

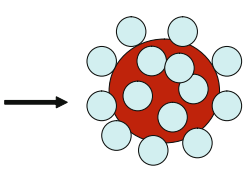

$\mathrm{n}_{\mathrm{m}}=20$

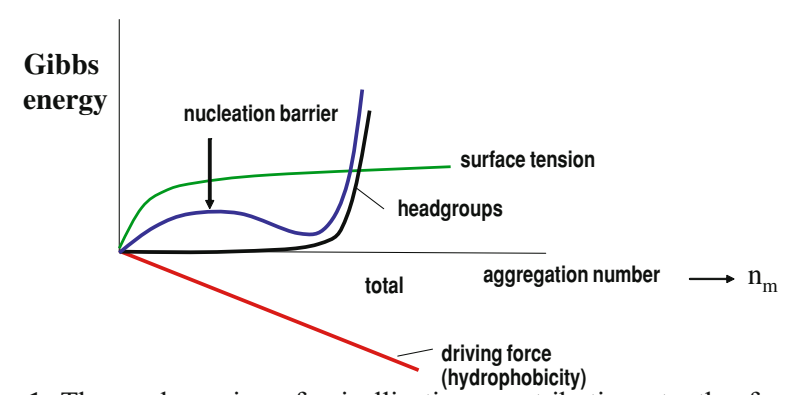

Fig. 1 Thermodynamics of micellization: contributions to the free energy as a function of aggregate size $n_{\mathrm{m}}$, showing driving force, surface tension correction, head group term. The sum curve of these three has a new minimum at the optimum aggregation number by the lyophilic moieties (head groups) of the molecules: the free energy increases as soon as these loose contact with the solvent by being 'buried' in the domain of insoluble parts. Hence, aggregate growth in all three spatial dimensions has to stop. This leaves two possibilities: growth has to stop altogether (we then get spherical micelles), or it must be limited to one dimension (cylindrical micelles) or two dimensions (lamellae) [4]. Due to the balance between lyophobic attraction and lyophilic repulsion, the free energy as a function of aggregation number (or of aggregation number per unit length or area) has a distinct minimum, which limits the extent to which size fluctuations can occur.

\section{Reversible supramolecular polymers}

The case of cylindrical micelles is interesting. Long cylindrical micelles share many properties with polymers because of their ability to undergo thermal shape (bending) fluctuations. The typical difference is, of course, that the covalent bonds in an ordinary polymer are extremely longlived, whereas these in a cylindrical micelle break and form rapidly at experimental time scales, which has consequences for their rheological behavior. Hence, these micelles can be seen as part of a special class of 'reversible supramolecular polymers' (RSP's), and quite some research has been devoted to them to understand their static and dynamic properties $[5,6]$. Cylindrical surfactant micelles are not the only possible kind of RSP; there are many more. For example, in almost all living creatures, the protein actin occurs; it forms filamentous assemblies that are part of the cytoskeleton, the structure responsible for the cell's mechanical performance [7]. In a biomimetic approach, we have recently prepared a new protein polymer which can be triggered to form very similar fibrils; this polymer will be discussed later in this review.

Another RSP subclass consists of bifunctional molecules that are capable to form reversible bonds with exactly two (complementary) partners. One recent example that attracted quite some attention is based upon the DNAinspired ureido-pyrimidinone (UPy) moiety, which dimerizes in non-aqueous systems due to hydrogen bonding [8]. A spacer carrying UPy moieties at both ends forms reversible chains, and new materials based upon this idea are now being developed. In our own group, we have recently explored the use of coordination chemistry for making reversible polymers with coordinated metal ions in the backbone. A pyridine ring carrying two carboxylic acid groups turned out to be a good ligand; a wide range of metal ions could form a bidentate complex with two such ligands, so that two such ligands connected back-to-back by a suitable spacer (denoted as ' $\mathrm{L}_{2} \mathrm{EO}_{n}$ ') should be able to form water-soluble coordination polymers (Fig. 2) [9]. 
Fig. $2 \mathrm{~L}_{2} \mathrm{EO}_{n}$ bisligand and doubly coordinated metal ion. The coordination center has a net charge of -2

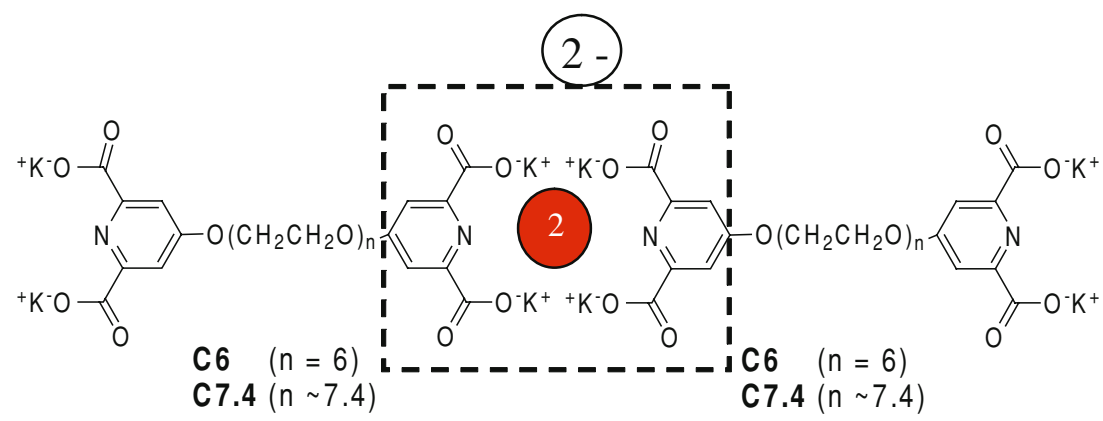

This turned out to be the case: at the proper (1:2) metal/ ligand ratio (e.g., with $\mathrm{Zn}$ as the coordinated metal), chains were found in the solution. However, small rings consisting of two bisligands and two metal ions were also detected in cases where the spacer had just the right length. Typically, low concentrations would then favor rings, whereas high concentrations would induce ring-opening polymerization of the rings to chains [10]. With large trivalent ions like neodymium $\left(\mathrm{Nd}^{3+}\right)$, triple coordination can occur, leading to branched polymers and reversible gels [11]. Clearly, RSP's are an intriguing class of spontaneously assembling materials that can be considered as colloids in their own right (Fig. 3).

\section{Complex coacervation}

However, one can do more. The ingredients discussed above can be used as the starting point for assembling new colloidal species with increased complexity. As explained, poor solubility and a stop mechanism are necessary, but also sufficient ingredients of a self-assembly process. When we consider solubility, we usually think of a single molecular species in a solvent: poor solubility then means that the solute segregates from the solvent. However, solubility may also be affected by attraction between two soluble species. What options do we have here?

Around 1930, Bungenberg de Jong investigated mixtures of oppositely charged macromolecules like gelatin and gum
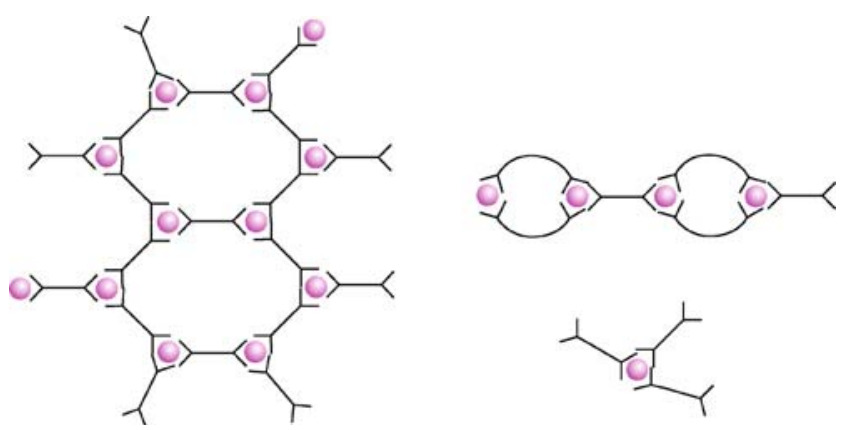

Fig. 3 Bisligands and $\mathrm{Nd}^{3+}$, forming structures with branches and rings. Lines represent the bisligand arabic, which were found to form complexes which could phase separate (at appropriate compositions) into a dilute watery phase and a concentrated fluid polymer complex phase [12]. He introduced the term 'complex coacervation' for this phenomenon. The formation of a complex between macromolecules carrying opposite charges can be seen as an ion-exchange process: ions in the overlapping double layers (the counterion clouds surrounding any macroion) are released when the oppositely charged polymers meet in a close encounter. This leads to an entropy increase determined by the relative change in local concentration experienced by the small ions, and to an additional energy decrease, if the average distance between opposite charges becomes smaller. Hence, unless the ionic strength is too high, macroions of opposite charge attract and tend to aggregate in a dense phase. Of course, bringing together macromolecules of like charge confines rather than releases the counterions, which leads to repulsion, as is very commonly known in colloid science [13]. Hence, what happens in mixtures of two oppositely charged macroions is therefore determined to a large extent by the charge stoichiometry, that is by how many charges are positive and how many are negative. A convenient way to express this is by the charge fraction $f_{i}$, the ratio between the number of (polymer) charges of a given sign ( $i$; plus or minus), and the total number of charges (plus and minus) on all polymers in the system. If $f$ is equal to 0.5 , the macroion charges are exactly matched and all counterions can be released; hence, there is no repulsion from small ions left and we expect phase separation. Of course, some left over small ions generate a repulsion that can suppress formation of a dense phase, but a certain number of them can be tolerated, so that phase separation can occur in a window of $f$ values around 0.5 . The width of this window decreases with ionic strength. Outside this window, at $f$ values closer to 0 or 1 , there are too many small ions that cannot be released and these prevent the system from phase separating. On the basis of these considerations one can draw a generic phase diagram, see Fig. 4.

The key issue is that we can have poor solubility with pairs of oppositely charged macromolecules. This might be employed to make films and capsules. The now very 


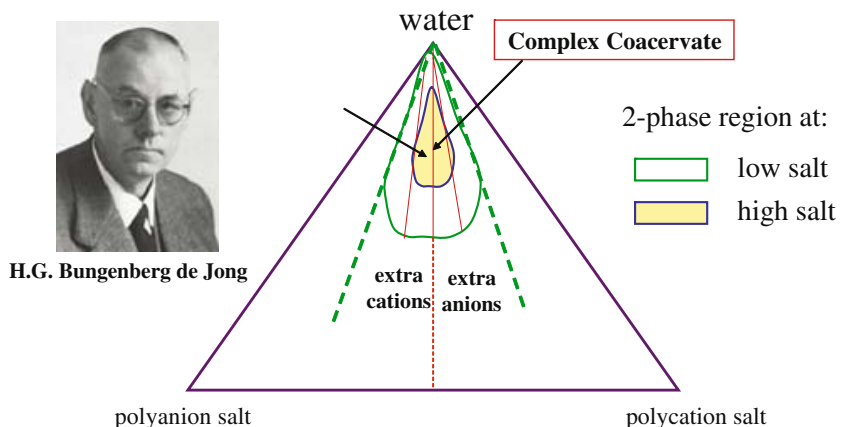

Fig. 4 Complex coacervation: generic phase diagram for aqueous mixtures of a polyanion and a polycation, at two different concentrations of added salt. Inset: H.C. Bungenberg de Jong

popular 'layer-by-layer' method [14] aims at doing so, and indeed works provided redissolution upon exposure to a polyelectrolyte solution is slow or even totally suppressed so that it cannot occur during a dipping cycle. Typically, polymers with strong ionic groups (e.g., sulfonates and quaternary ammonium groups) are good at this, and backbone hydrophobicity also helps. In other cases, however, redissolution does occur [15].

\section{Complex coacervate micelles}

What about a stop mechanism? This is straightforward: an uncharged block or graft added to a charged polymer can very well fulfill this role, provided it has no tendency to enter the dense complex phase. Given the general tendency of polymers to avoid mixing unless there is a specific attraction, this is very unlikely to happen. Hence, we can make micelles with a pair of oppositely charged macromolecules, at least one of which carries a water-soluble, non-ionic block [16]. Such micelles are now known for about 12 years, in many variations. They are usually spherical, having radii varying between 10 and $30 \mathrm{~nm}$, and typically consist of a relatively dense core which contains the insoluble polyelectrolyte complex, surrounded by a more dilute corona of swollen neutral and hydrophilic chains; we denote them accordingly as Complex Coacervate Core Micelles or C3M's. Other terms used are 'PIC micelles' (polyion complex micelles) or 'BIC's' ('block ionomer complexes'). In several respects, they are very similar to the more familiar micelles formed by amphiphilic diblock copolymers such as, e.g., poly ethylene oxide- $b$-polystyrene. New features are, however, that they are sensitive to solution parameters that affect the ionic complex, such as salt concentration and $\mathrm{pH}$, and that they often respond reversibly to changes in polymer composition: they appear nearstoichiometric conditions, but disappear upon leaving the near-stoichiometric range, just like the complex coacervates do. Micelles of this kind are highly interesting candidates for targeted drug delivery to tumors, since they have just the right size to benefit of the Enhanced Permeability and Retention (EPR) effect that occurs in tumor tissue due to the leaking of blood vessels, and they can be loaded with various anticancer drugs [17].

\section{Janus micelles}

From a colloid science viewpoint, one may now ask the question: to what extent do these systems enrich our science, by introducing new types of colloids and posing new questions? As our first example, we discuss particles with mixed coronas. The very fact that it takes two species rather than one to make these micelles allows us to choose a pair of dissimilar water-soluble blocks for the corona. Since, generally, dissimilar polymers tend to avoid mixing, this generates micelles which force chains which would otherwise stay apart to 'live together' due to the attraction between the charged blocks they are connected to. Hence, the corona tries to phase separate without destroying the micelle, and the simplest way it can do this is to become a bipolar particle, with the different corona chains sticking out on either side. Such micelles would be the first instance of reversible (self-assembled) bipolar particles; we denote them as 'Janus micelles' after the double-faced Roman deity Janus. As the reader will appreciate, proving the 'Janus' character is not a trivial task since typical methods for obtaining the relevant structural information (e.g., scattering) tend to be insufficiently sensitive at the nanolevel. However, we succeeded; in Figs. 5-7, we present the evidence which convinced us [18].

The polymers used were polyacrylate- $b$-polyacryl amide $\left(\mathrm{PAA}_{42}-b-\mathrm{PAAm}_{417}\right)$ and poly ethylene oxide- $b$-poly- $N$ -

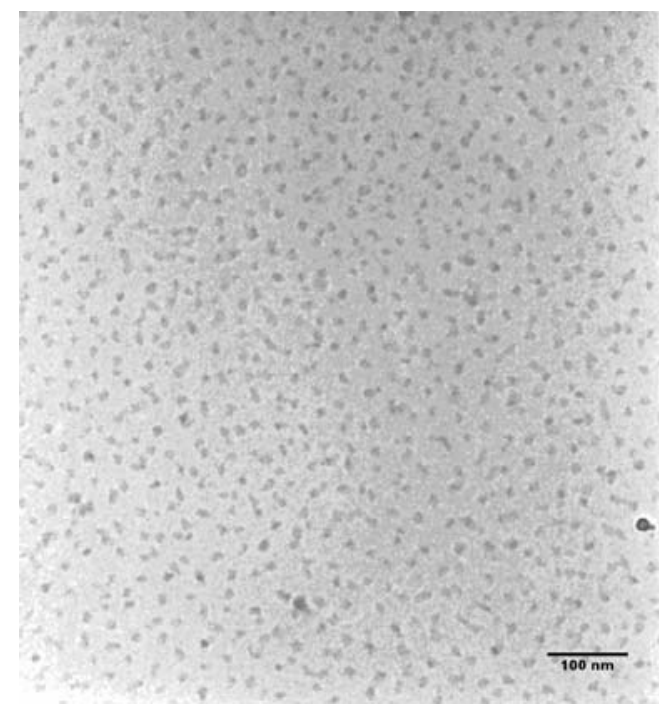

Fig. 5 Janus micelles: cryo-TEM, showing non-spherical objects. Scale bar: $100 \mathrm{~nm}$ 


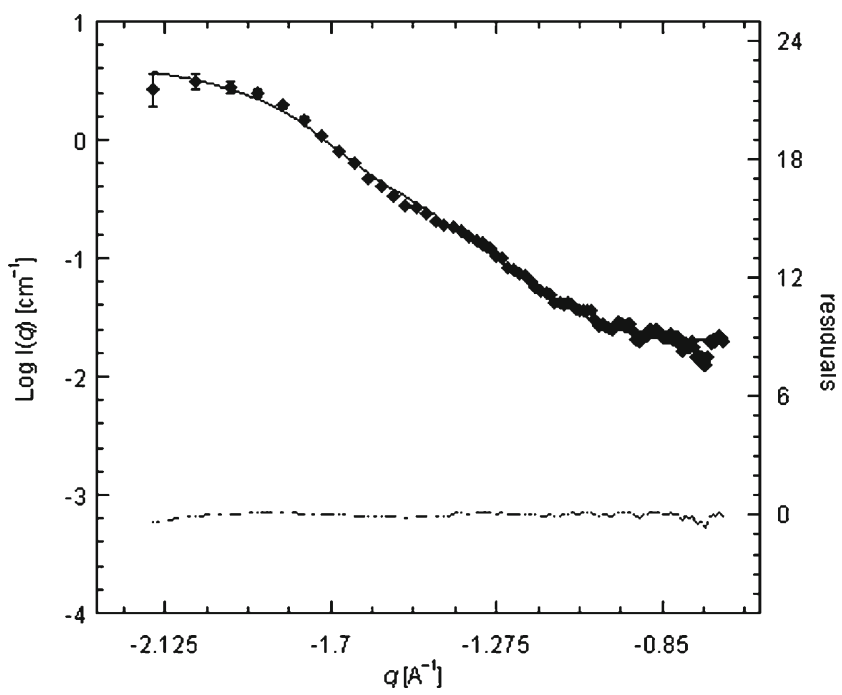

Fig. 6 Janus micelles: SANS data (diamonds: data; curve: form factor fit; small dots: residuals)

methyl-2-vinylpyridinium iodide ( $\left.\mathrm{P}^{2} \mathrm{MVP}_{42}-b-\mathrm{PEO}_{446}\right)$, mixed so that $f_{=} 0.5$, in dilute aqueous solution. First, cryo-TEM images (Fig. 5) show a mixture of circular and elliptical objects, as if one is looking at a collection of randomly oriented discs. Usually, the corona is invisible in such pictures so that, probably, we deal with disc-shaped cores.

This is confirmed by SANS data (Fig. 6). A fit with a shape factor for ellipsoids with main axes of 18 and $2.5 \mathrm{~nm}$, respectively, is the only one giving satisfactory agreement (see residuals), without having to assume very exotic polydispersities which are not seen in cryo-TEM. Moreover, the sizes found closely match those of the images.

So far, this only tells us we have a non-spherical core shape, which would be very unlikely without some sort of symmetry breaking tendency. A more direct piece of evidence that the corona is not mixed comes from NMRNOESY experiments. In such experiments, a particular nuclear resonance can be observed by irradiation of a nucleus which is chemically different but spatially close: magnetization is then transferred by coupling between the spins. As a result, the 2D NOESY spectrum of true mixtures of two polymers shows off-diagonal peaks, whereas such peaks would be absent for a demixed corona. Both cases do indeed occur (Fig. 7): polyacryl amide and poly glyceryl methacrylate mix (Fig. 7, left diagram), but poly acrylamide and poly (ethylene oxide) do not (Fig. 7, right diagram), which strongly supports the Janus hypothesis.

Finally, the alleged Janus system shows depolarization of scattered polarized light. This not only betrays a certain amount of optical anisotropy, but also allows to estimate the rotational diffusion coefficient. The data suggest an ellipsoidal shape, but much larger than that is seen by SANS. The way this can be accounted for is that each 'face' of the disc-like core carries a 'lobe' of swollen corona chains, one side PEO, the other side PAAm. Hence, our Janus particles can be represented by the sketch in Fig. 8.

Theoretical modeling also shows that a double-lobe structure is quite likely to appear (Fig. 9) provided the repulsion (represented by a Flory-Huggins interaction parameter) is large enough [19].

As is clear from the example just discussed, the very fact that C3M's consist of two oppositely charged species opens new options. A further step is that one can choose one of the 'lobes' of a Janus micelle to be a temperature-sensitive polymer such as, e.g., PNIPAM (poly $N$-isopropyl acryl-
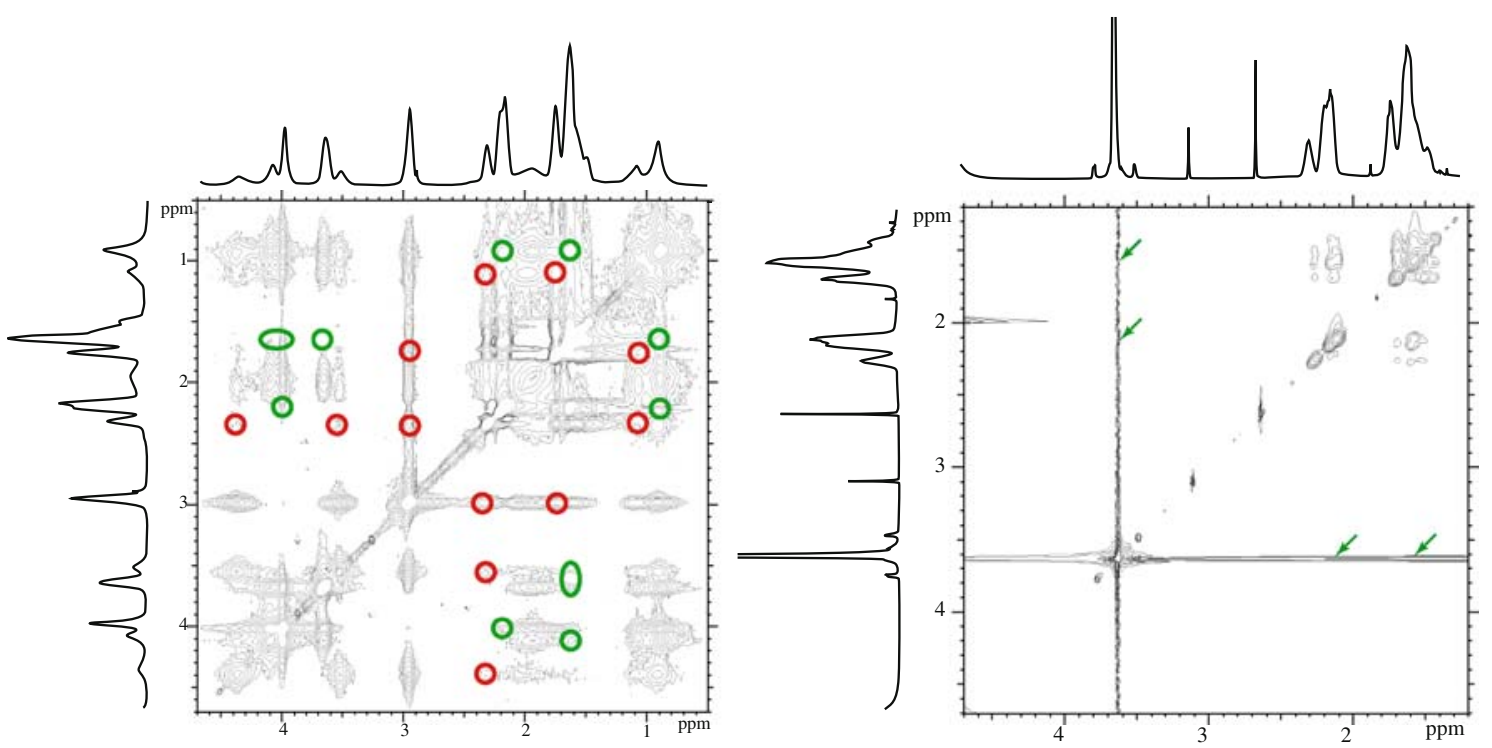

Fig. 7 Janus micelles: NMR-NOESY spectra. Left diagram: polyacryl amide+poly glyceryl methacrylate corona (miscible); circles denote cross peaks due to intermolecular contact; right diagram: poly acrylamide+poly (ethylene oxide) corona (demixed) 


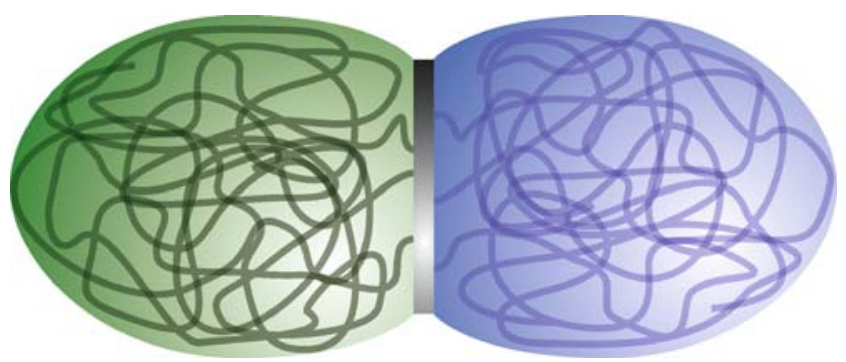

Fig. 8 Janus micelle structure (cartoon) as inferred from data. Grey: complex coacervate core; blue: poly (ethylene oxide); green: poly acrylamide

amide) or PDEGMA (poly diethylene glycol methacrylate). Upon increasing the temperature above the lower critical solubility temperature (LCST), the Janus micelle will have one side in a poor solvent condition, leading to collapse and attraction. As a result, several micelles will cluster to form a large particle, but, again, the presence of the solvated sides will prevent macroscopic phase separation. Hence, some sort of 'supermicelle' will appear as long as the temperature exceeds the LCST. This is precisely what we observe (Fig. 10) with micelles consisting of PAA- $b$-PAAm and PDMAEMA- $b$-PDEGMA (this latter block has an LCST of about $35{ }^{\circ} \mathrm{C}$ ), but also with PAA- $b$-PNIPAM and PEO- $b$ PVPy (PNIPAM has an LCST of about $32{ }^{\circ} \mathrm{C}$ ).

As to the dynamics of formation, there is a fundamental difference between two-component and one-component micelles. As is often the case in a colloid preparation method, one starts out with a soluble precursor that is driven into a colloidal state by a change in solubility due to, e.g., chemical reaction, temperature change or solvent change. Polymer micelles are usually prepared by a 'solvent quench', i.e., a rapid change in solvent composition. When this is done with a conventional amphiphilic copolymer, the one block which becomes insoluble will collapse (which it can do without any other polymer molecule around) after which it aggregates into a multimolecular particle. Only the rate of the latter process is concentration dependent; in dilute solutions, the unimolecular collapse process will always be finished before any aggregation takes place. As a result, the formation of micelles proceeds rather smoothly, even though quite some shape variation is possible [20]. With C3M's this is completely different. Here, the first step is always intermolecular, no unimolecular collapse is possible. What then happens depends strongly on composition. At compositions around $f=0.5$, the random encounters between molecules of opposite charge initially leads to a dense, insoluble network that can subsequently rearrange into micelles. The rearrangement process requires the multiple breaking and forming of ions pairs which can be very slow due to the associated energy barriers [21], or may be even blocked altogether. At compositions far from $f=$ 0.5 , the build up of multimolecular species is much more gradual. Hence, the rate at which components are mixed has great consequences for the kinetic pathway taken by the system and, depending on relaxation rates, may sometimes lead to equilibrated structures, sometimes to frozen particles [22]. One can therefore say that these systems take an intermediate position between the 'lyophobic' and 'lyophilic' classes traditionally distinguished in colloid science.

\section{Hierarchical self-assembly}

What other options do we have? A diblock copolymer is very effective in providing a stop mechanism. Hence, this ingredient cannot be missed. However, for the other ingredient(s) one has quite some freedom. Simple polyelectrolytes of varying length have been used, but also proteins (enzymes) [23], ionic surfactants [24], and inor-
Fig. 9 Symmetry breaking in a micelle with a mixed corona, as modeled by means of selfconsistent field calculations [19] imposing a non-deformable spherical core. The $3 D$ picture presents monomer densities (vertical) as a function of position in the $x, y$ plane cutting through the particle center

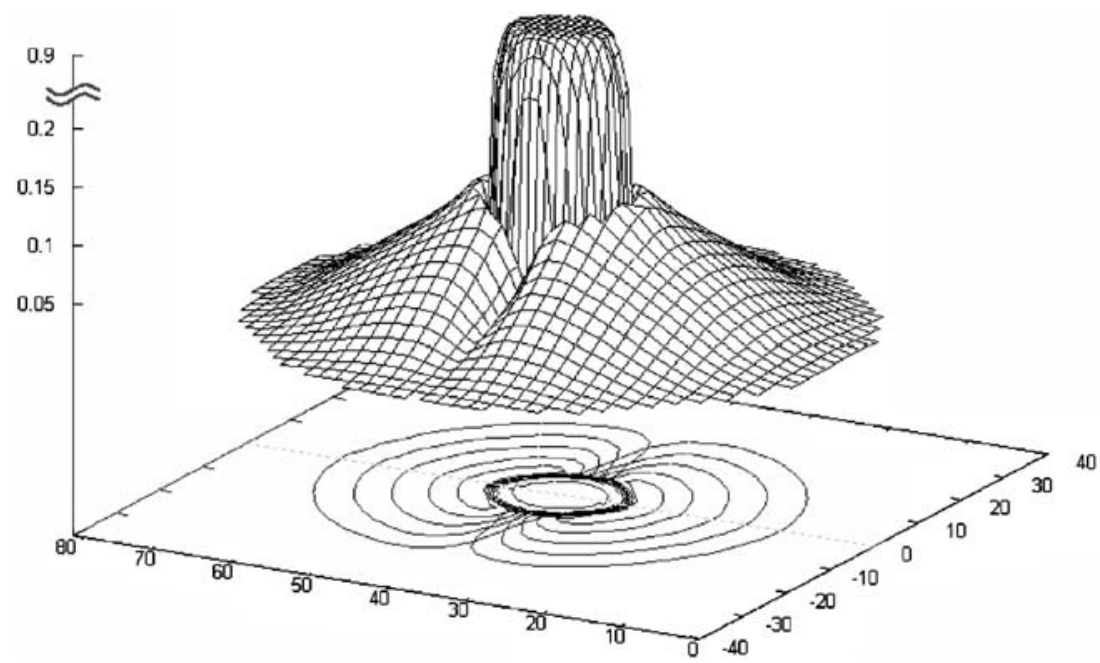



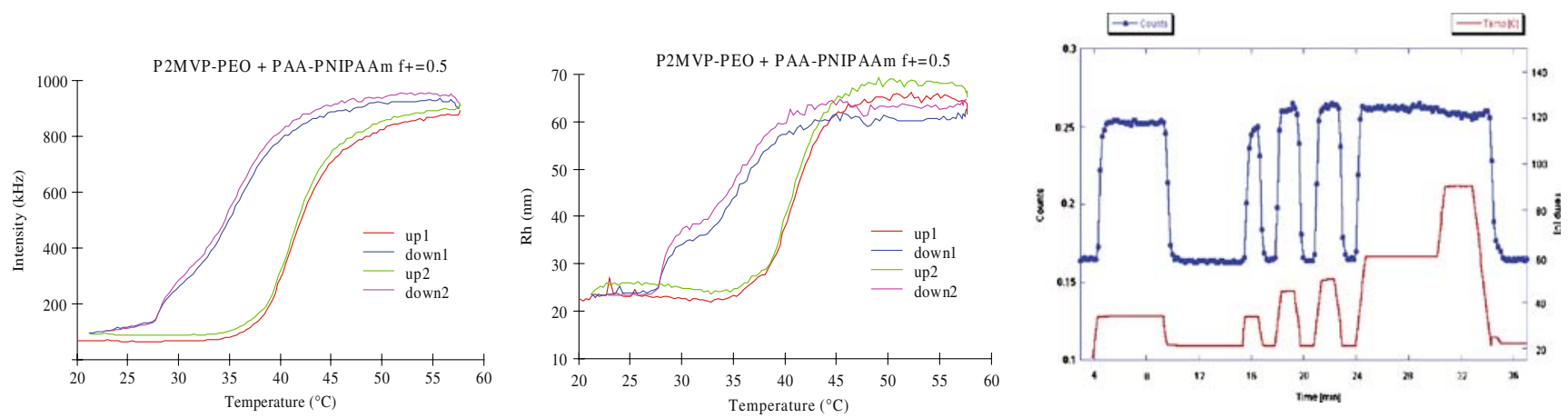

Fig. 10 Aggregation of Janus micelles upon temperature increase. Left: PAA- $b$-PNIPAM+PEO- $b$-PVPy, particle mass and particle hydrodynamic radius upon increasing and decreasing temperature, from 20 to $55^{\circ} \mathrm{C}$. The particle mass increases more than tenfold, the size by a factor of about 2.5. Hysteresis is due to (too) rapid heating

ganic particles have been successfully incorporated. Systems composed of an ionic surfactant and diblock copolymers deserve some attention, since they can be considered as 'micelles within micelles' that is, an example of hierarchical structures. Low MW surfactant micelles are very dynamic, and therefore, the compound micelles based on them are equilibrium systems; their structure is entirely independent of sample history.

Another example of a hierarchical nanostructure is the $\mathrm{C} 3 \mathrm{M}$ formed between a diblock copolymer and a charged water-soluble RSP. We have recently introduced such C3M's by using a negatively charged coordination polymer formed by the $\mathrm{L}_{2} \mathrm{EO}_{n}$ bisligands and metal ions, with diblocks consisting of PEO and positively charged P2MVP $[25,26]$. In this case, it is the coordination compound which polymerizes (self-assembles) upon forming the complex with P2MVP, even though it is present in the form of small rings in the starting solution. The coordination polymer introduces metal ions into the micellar core $\left(\mathrm{Zn}^{2+}, \mathrm{Ni}^{2+}, \mathrm{Fe}^{2+}, \mathrm{Fe}^{3+}, \mathrm{Nd}^{3+}, \ldots\right)$ that endow it with high electron densities, rendering them nicely visible in EM pictures (Fig. 11) as well as with magnetic properties for appropriate metals $(\mathrm{Fe}, \mathrm{Co})$.

The fact that the coordination compound polymerizes reversibly manifests itself in other ways. One is by the fact that off-stoichiometric compositions behave in an asymmetric fashion. At $f+>0.5$, soluble complexes exist because excess PVPy provides the repulsive interaction which prevents mutual aggregation of the block copolymers. At $f$ $+<0.5$, however, the excess coordination compound usually cannot polymerize beyond the dimer state (which is insufficient to stabilize small species) unless the concentration is high enough. Coordination complexes with large trivalent ions like, e.g., $\mathrm{Nd}^{3+}$, are more potent stabilizers, as they can form trivalent (L3Me) complexes with a single ion, leading to polymers with rings and branches which are much less likely to break up under thermal motion and and cooling; very slow temperature changes $\left(<0.03{ }^{\circ} \mathrm{C} / \mathrm{min}\right)$ produce a reversible curve. Right: changes in scattered intensity (neutrons) upon changing temperature between 20 and $90{ }^{\circ} \mathrm{C}$. Bottom curve: temperature profile; top curve: response of the scattered intensity

therefore persist down to significantly lower concentrations [27]. This can be clearly seen in Fig. 12 which compares C3M's with $\mathrm{Zn}$ and Nd. The implication is that in a solution of ring shaped $\mathrm{L}_{2} \mathrm{EO}_{n} / \mathrm{Zn}$, any added PEO-PVPy will very soon produce colloidal C3M's, whereas with $\mathrm{L}_{2} \mathrm{EO}_{n} / \mathrm{Nd}$ complexes one needs to approach $f+=0.5$ much closer before this happens.

Another proof of reversibility is the fact that a CMC can be measured for these micelles. As the cohesive strength of the cores is highly salt-sensitive, we should expect a saltdependent CMC, and we do find that, as can be seen in Fig. 13.

\section{Supramolecular surface forces}

A central concern of colloid science is with forces between lyophobic colloidal particles. These can be electrodynamic

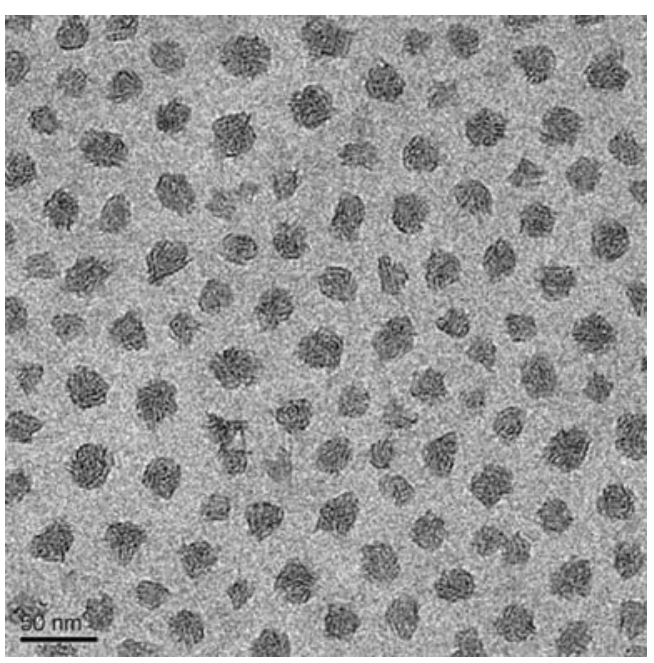

Fig. $11 \mathrm{C} 3 \mathrm{M}$ nanoparticles formed from PEO- $b$-P2MVP and $\mathrm{Fe}^{2+}$ coordinated by $\mathrm{L}_{2} \mathrm{EO}_{n}$. The cores of the particles are visible; note their irregular shapes. Scale bar: $50 \mathrm{~nm}$ 


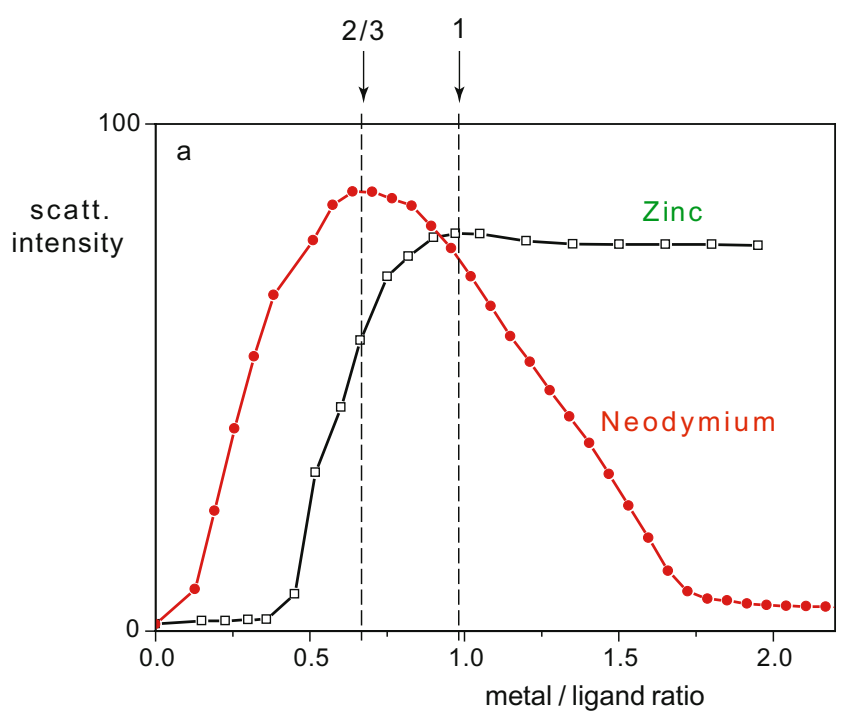

Fig. 12 Comparison of C3M's made with $\mathrm{L}_{2} \mathrm{EO}_{n}$ and $\mathrm{Zn}^{2+}$ and with $\mathrm{L}_{2} \mathrm{EO}_{n}$ and $\mathrm{Nd}^{3+}$. The $\mathrm{Nd}$ complex can stabilize small soluble particles, so that the micelles disappear at high metal concentrations, but the $\mathrm{Zn}$ complex cannot, so that the micelles persist at high metal content

in origin and arise from the body of the particles (van der Waals forces, dispersion forces), but quite often they must be assigned to properties of the particle/medium interface (true surface forces). As such, they are the result of all the interactions between the particle and the surrounding medium, including various solutes. The well-known double-layer interaction is an example that has been studied at length [28]; and also steric and depletion forces induced by soluble polymers have received much attention [29].

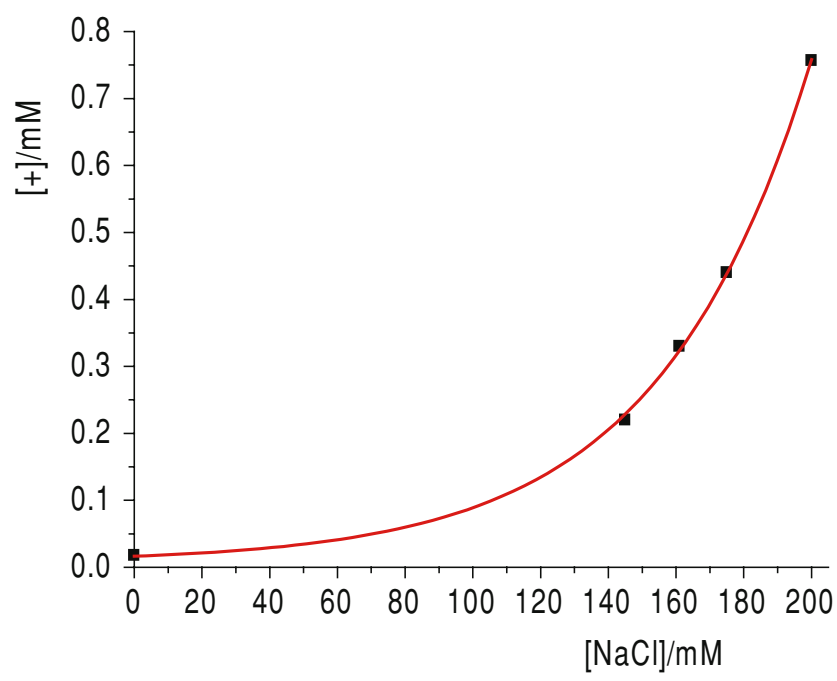

Fig. $13 \mathrm{CMC}$ (expressed as $\mathrm{mM}$ of cationic polymer in the system) as a function of ionic strength $(\mathrm{NaCl})$ in $\mathrm{mM}$
However, there are many more cases where subtle processes in solution show up as colloidal interactions or surface forces. We consider some cases where selfassembling objects are involved.

One example which has been discussed from a general theoretical viewpoint is that of RSP's between surfaces [30]. RSP's come in two classes: 'non-directional' and 'directional'. The first class forms structures which possess inversion symmetry: the chains do not have a 'head' and a 'tail' or a ' + ' and '-' end. The second class does, because all the units have head and tail. The RSP's based on coordination complexes clearly belong to the former class; an example of the former would be a single-stranded DNA chain the ends of which (when read in the same direction) are complementary. As can be shown, the non-directional RSP's, when endattached to identical surfaces, always generate attraction, whereas the non-directional ones can generate repulsion. Compatible mixtures of the two can even generate nonmonotonic interactions. One example of attraction in the case of a non-directional RSP has been recently reported [31], but the 'directional' case has not been realized yet.

A second example is that of solutions of self-assembling species interacting with a solid/liquid interface [32]. A third example is a solution of self-assembling species close to a phase separation boundary. Here, the presence of a surface may induce a 'precursor' of the incipient phase when it prefers solute over solvent. If this occurs for liquid/liquid phase separation, one could refer to it as capillary condensation, and the ensuing force is a capillary force. A beautiful example was recently reported [33] and it seems that many more examples lie awaiting us in multicomponent complex fluids. They definitely deserve investigation,

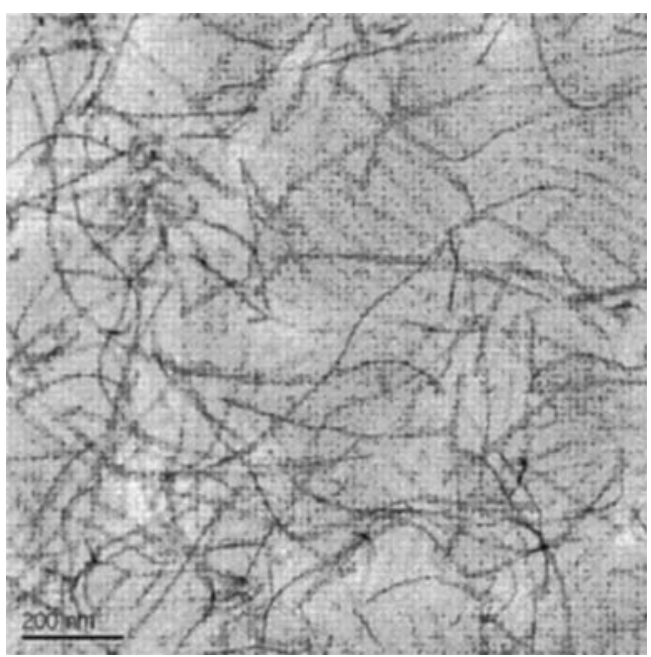

Fig. 14 Tape-like assemblies resulting from mutually induced polymerization of the positively charged protein $\mathrm{CS}^{\mathrm{H}} \mathrm{S}^{\mathrm{H}} \mathrm{C}$ with the negatively charged coordination compound $\mathrm{L}_{2} \mathrm{EO}_{n} / \mathrm{Zn}$. Note the difference between narrow, high-contrast parts (edge-on view) and wider, low-contrast parts (top view) of the tapes. Scale bar: $200 \mathrm{~nm}$ 
particularly from a dynamic point of view. Innovations from the side of synthetic chemistry will bring in new compounds that might induce such surface forces.

\section{Where can we go from here?}

Let us discuss some ideas. Copolymers with a diblock structure tend to primarily form micelles, but copolymers with a triblock sequence (sometimes called telechelics) can do more. In particular, they can make networks of micelles. In the latter category we find, e.g., the associative thickeners based on polyethers (PEO) with insoluble (alkyl) end groups widely applied in water-based paints. Following up on this, one would expect that telechelics with charged end blocks would also be able to form similar networks in the presence of a stoichiometric amount of oppositely charged macroions. Remarkably, no literature reports on such networks can be found in the literature so that we have started an investigation in this direction.

A second example is the combination of two oppositely charged RSP's. We already discussed an example of a negatively charged coordination polymer. Recently, we studied a positively charged protein polymer with a triblock structure (labeled $\mathrm{CS}^{\mathrm{H}} \mathrm{S}^{\mathrm{H}} \mathrm{C}$ ) that self-assembled into linear structures (fibrils) whenever its charge was screened. This is a designed protein with a block-like sequence; its end blocks are hydrophilic and swell in water, whereas its middle block can form a so-called 'beta roll' which exposes hydrophobic faces and then polymerizes by hydrophobicity driven stacking. In a solution of neutral $\mathrm{pH}$, the protein simply forms a molecular solution, but upon adding a dilute solution of the $\mathrm{L}_{2} \mathrm{EO}_{n} / \mathrm{Zn}$ compound, both the protein and the coordination RSP polymerized together, forming a tapelike structure that can be very well seen in cryo-TEM pictures (Fig. 14). Clearly, combining two (or more) ingredients that co-assemble rather than just self-assembly leads to very interesting new nano-objects and materials, and is a challenging direction for colloid science.

\section{Concluding remarks}

Colloid Science, with its roots in Physical Chemistry, and Supramolecular Chemistry, with its firm basis of synthetic organic and organometallic chemistry can mutually benefit a lot. The appearance of new structures and ways to tune and manipulate structures enriches both fields. Promising developments are to be found also in the realm of biopolymers, where the repertoire of possible conformations and internal interactions is so much larger than that for most synthetic polymers, and rules of thumb developed for simple polymers do not apply. Hence, nanoassemblies of biopolymers (and hybrids of these with synthetic compounds) are a great target for future work.

Open Access This article is distributed under the terms of the Creative Commons Attribution Noncommercial License which permits any noncommercial use, distribution, and reproduction in any medium, provided the original author(s) and source are credited.

\section{References}

1. Lehn JM (1995) Supramolecular Chemistry: Concepts and Perspectives VCH, Weinheim

2. McBain JW (1913) Trans Faraday Soc 9:99

3. Overbeek JThG (1952) In: Kruyt HR (ed) Colloid science. vol. I. Elsevier, Amsterdam

4. Gelbart WM, Ben-Shaul A Roux D, eds (1994) Micelles, Membranes, Microemulsions and Monolayers Springer, NY

5. Cates ME, Candau SJ (1990) J Phys Condens. Matter 2:6869-6892

6. Knoben W, Besseling NAM, Cohen Stuart MA (2007) J Chem Phys 126:24907

7. Van der Gucht J, Paluch E, Sykes C, Plastino J (2005) Proc Natl Acad Sci 102:7847-7852

8. Hirschberg JHKK, Beijer FH, Van Aert HA, Magusim PCMM, Sijbesma RP, Meijer EW (1999) Macromolecules 32:2696-2705

9. Vermonden T, Van der Gucht J, De Waard P, Marcelis ATM, Besseling NAM, Sudhölter EJR, Fleer GJ, Cohen Stuart MA (2003) Macromolecules 36:7035-7044

10. Vermonden T, De Vos W, Marcelis ATM (2004) Eur J Inorg Chem $14: 2847-2852$

11. Vermonden T, Van Steenbergen MJ, Besseling NAM, Marcelis ATM, Hennink WE, Sudhölter EJR, Cohen Stuart MA (2004) J Am Chem Soc 126:15802-15808

12. Bungenberg de Jong HG, Kruyt HR (1929) Proc Acad Sci Amsterdam 32:849

13. Verwey EJW, Overbeek JThG (1948) Theory of the stability of lyophobic colloids. The interaction of sol particles having an electric double layer, Ch. V.. Elsevier, Amsterdam

14. Decher G (1997) Science 277:1232-1237

15. Kovacevic D, Van der Burgh S, De Keizer A, Cohen Stuart MA (2002) Langmuir 18:5607-12

16. Cohen Stuart MA, Hofs PS, Voets IK, De Keizer A (2005) Curr Op Colloid Interface Sci. 10:30-36

17. Cabral H, Nishiyama N, Kataoka K (2007) J Controlled Release 121:146-155

18. Voets IK, De Keizer A, De Waard P, Frederik PM, Bomans PHH, Schmalz H, Walther A, King SM, Leermakers FAM, Cohen Stuart MA (2006) Angew Chem Int Ed 45:6673-6676

19. Charlaganov M, Borisov O, Leermakers FAM, Macromolecules, in press (ma-2008-00130q) DOI 10.1021/ma800130g

20. Shen HW, Eisenberg A (2000) Macromolecules 33:2561-2572

21. Cohen Stuart MA, Besseling NAM, Fokkink RG (1998) Langmuir 14:6846-6849

22. Voets IK, De Keizer A, Cohen Stuart MA, Justynka J Schlaad H (2007) Macromolecules 40:2158-2164

23. Lindhoud S, De Vries R, Norde W, Cohen Stuart MA (2007) Biomacromolecules 8:2219-2227

24. Berret JF, Oberdisse (2004) J Physica B Condens Matter 350:204-206

25. Yan Y, Besseling NAM, De Keizer A, Marcelis ATM, Drechsler M, Cohen Stuart MA (2007) Angew Chem Int Ed 46:1807-1809

26. Yan Y, Besseling NAM, De Keizer A, Drechsler M, Fokkink R, Cohen Stuart MA (2007) Phys Chem B 111:11662-11669

27. Yan Y, Besseling NAM, De Keizer A, Cohen Stuart MA (2007) J Phys Chem B 111:5811-5818 
28. Lyklema J (2005) Fundamentals of interface and colloid science IV, Ch. 3. Academic, London

29. Fleer GJ, Cohen Stuart MA, Leermakers FAM (2005) Fundamentals of Interface and Colloid Science V, Ch. 1. Academic, London

30. Van der Gucht J, Besseling NAM, Cohen Stuart MA (2002) J Am Chem Soc 124:6202-6205
31. Knoben W, Besseling NAM, Cohen Stuart MA (2007) Langmuir 23:6095-6105

32. Leermakers FAM, Koopal LK, Lokar WJ, Ducker WA (2005) Langmuir 21:11534-11545

33. Sprakel J, Besseling NAM, Leermakers FAM, Cohen Stuart MA (2007) Phys Rev Lett 99:104504 